Correspondence
Sharon Chen
Sharon.Chen@swahs.health.nsw.
gov.au

Received 1 December 2009 Accepted 23 January 2010

\section{Reverse line blot hybridization and DNA sequencing studies of the 16S-23S rRNA gene intergenic spacer regions of five emerging pathogenic Nocardia species}

Xiaoyan Wang, ${ }^{1,2,3} \dagger$ Meng Xiao, ${ }^{4} \dagger$ Fanrong Kong ${ }^{3} \dagger$ Vitali Sintchenko, ${ }^{3}$ Huiping Wang, ${ }^{5}$ Bin Wang, ${ }^{6}$ Shi Lian, ${ }^{1}$ Tania Sorrell ${ }^{3}$ and Sharon Chen $^{3}$

${ }^{1}$ Department of Dermatology and Venerology, Xuan Wu Hospital, Capital Medical University, Beijing, PR China

${ }^{2}$ Department of Dermatology and Venerology, The Affiliated Hospital of Inner Mongolia Medical College, Hohhot, PR China

${ }^{3}$ Centre for Infectious Diseases and Microbiology, The University of Sydney, Westmead Hospital, Westmead, New South Wales, Australia

${ }^{4}$ Life Science College, Peking University, Beijing, PR China

${ }^{5}$ Department of Dermatology, Tianjin Medical University General Hospital, Tianjin, PR China

${ }^{6}$ Retroviral Genetics Laboratory, Centre for Virus Research, Westmead Millennium Institute, The University of Sydney, Westmead, New South Wales, Australia

The objective of this study was to examine DNA sequence polymorphisms in the 16S-23S rRNA gene intergenic spacer (ITS) regions of five emerging pathogenic Nocardia species: Nocardia beijingensis, Nocardia blacklockiae, Nocardia thailandica, Nocardia elegans and Nocardia vinacea. A set of six isolates belonging to the species of interest and 135 isolates belonging to other Nocardia species was studied. A PCR-based reverse line blot (RLB) hybridization assay incorporating species- or intraspecies ITS rRNA gene operon-specific probes was then developed for species identification. Substantial intraspecies sequence variation among different ITS operons was identified. Four sequence types of $N$. thailandica, eight sequence types of $N$. beijingensis (four types for each of two strains) and five sequence types of $N$. blacklockiae, $N$. elegans and $N$. vinacea were found. The results represent the first evidence of ITS sequence heterogeneity in emerging species of Nocardia. By incorporating species/operon-specific probes into a RLB assay, unique RLB patterns were identified for each of the species and every sequence type. The PCR/RLB assay demonstrated high specificity and showed promise in both the identification and genotyping of Nocardia species. More detailed studies of the polymorphism within the ITS locus may further advance our capacity to reliably identify and subtype medically important Nocardia species.
†These authors contributed equally to this work.

Abbreviations: ITS, (16S-23S rRNA gene) intergenic spacer; RLB, reverse line blot.

The GenBank/EMBL/DDBJ accession numbers for the 16S-23S rRNA gene intergenic spacer region sequences of the $N$. beijingensis, $N$. blacklockiae, $N$. elegans, $N$. thailandica and $N$. vinacea isolates are G0853471-G0853497 (Table 1).

Supplementary information is available with the online version of this paper.

\section{INTRODUCTION}

Nocardia species cause infections ranging from localized skin and lung infection to disseminated disease (Saubolle \& Sussland, 2003). About 90 species have now been described of which about half have been implicated in human disease (http://www.bacterio.cict.fr/n/nocardia.html). Speciation of clinical isolates is essential for determining associated disease manifestations and to predict antimicrobial susceptibility.

16S rRNA gene sequencing is the most frequently used method for definitive species identification of nocardiae. However, it may be unable to distinguish closely related 
species due to insufficient interspecies polymorphisms (Brown-Elliott et al., 2006; Kong et al., 2009; Roth et al., 2003); misidentifications may also occur due to multiple, but different, 16S rRNA gene copies (Conville \& Witebsky, 2007). Polymorphisms within alternative targets including the genes encoding Nocardia $65 \mathrm{kDa}$ heat-shock protein (hsp65), essential secretory protein A (secAl) and gyrase B (gyrB) have been reported to improve species identification (Conville et al., 2006; Rodriguez-Nava et al., 2006; Takeda et al., 2010). A further candidate target is the 16S-23S rRNA gene intergenic spacer (ITS) region, which contains variable signatures for accurate interspecies discrimination that have been exploited for identification of a number of bacteria (Park et al., 2005; Xiong et al., 2006; Su et al., 2009). However, their utility in providing species identification of Nocardia is not well defined.

Examination of ITS sequence entries in the GenBank database revealed Nocardia sequences for approximately a dozen of the more common species (e.g. Nocardia cyriacigeorgica, Nocardia farcinica, Nocardia nova; http:// www.ncbi.nlm.nih.gov). However, few data are available for uncommon but emerging species: specifically, we were unable, at the time of study, to locate sequences for Nocardia beijingensis, Nocardia blacklockiae, Nocardia elegans, Nocardia thailandica and Nocardia vinacea, five species recently recognized as causes of pulmonary, subcutaneous and bloodstream infection (Kageyama et al., 2004a, b, 2005; Conville et al., 2008; Yassin \& Brenner, 2005; Luong et al., 2009). Alignment of current GenBank sequences indicates multiple ( three to five) different ITS copies (operons) to be present in a single isolate. Thus a direct sequencing approach may not allow discrimination between species (Gurtler \& Stanisich, 1996).

We previously developed a method to identify bacteria using PCR followed by hybridization with species-specific oligonucleotide probes in a reverse line blot (RLB) assay (Wang et al., 2008; Xiong et al., 2006). During the evaluation of such an RLB assay employing 16S rRNA gene-targeted probes to identify the more common Nocardia species, we noted that ITS sequence polymorphisms were more discriminatory for the identification of two species, N. farcinica and Nocardia paucivorans (Xiao et al., 2010). Because 16S-23S rRNA gene ITS sequences were not publicly available for $N$. beijingensis, $N$. blacklockiae, $N$. elegans, $N$. thailandica and $N$. vinacea, in the present study, we sought to (i) describe sequence polymorphisms in the ITS locus for these species through cloning and sequencing of the ITS regions, and (ii) develop an ITStargeted RLB assay based on gene polymorphisms identified in (i) for the identification of these five species.

\section{METHODS}

Nocardia organisms and DNA extraction. A total of 141 clinical Nocardia isolates were obtained from the culture collection at the Clinical Mycology Laboratory, Centre for Infectious Diseases and Microbiology, Westmead Hospital, Sydney. These comprised six isolates belonging to the five species of interest (two $N$. beijingensis isolates and one strain each of $N$. blacklockiae, N. elegans, N. thailandica and N. vinacea; Table 1) and 135 isolates of other Nocardia species (ie. non-target species strains; see Supplementary Table S1 in JMM Online for details). All strains were examined by the RLB assay. In all cases, isolates were identified using standard phenotypic-based methods (McNeil \& Brown, 1994) and species identification was confirmed by partial ( $5^{\prime}$-end $606 \mathrm{bp}$ fragment) $16 \mathrm{~S}$ rRNA gene sequencing based on comparison of sequences with those of the 'reference' or type strains of their respective species in the GenBank database as previously described (Kong et al., 2009; Xiao et al., 2010). The isolates were cultured from separate patients with no epidemiological links.

Organisms were cultured in brain heart infusion broth (Amyl Media) for 3-10 days at $37{ }^{\circ} \mathrm{C}$ in air prior to DNA extraction (McNeil \& Brown, 1994). DNA extraction was carried out as previously reported (Kong et al., 2009).

Cloning of the ITS region. Cloning of the ITS locus of the six study isolates was performed as described below to minimize the impact of ITS sequence heterogeneity due to multiple ITS copies or operons within the one isolate.

PCR of the ITS region. The primer pair $16 \mathrm{Sf}$ and $23 \mathrm{Sr}$ (Table 2) was used to amplify the Nocardia ITS region. Primers were designed 'in house' and synthesized at Beijing AuGCT Biotechnology. PCR amplification was carried out as described previously (Xiao et al., 2010). The expected PCR product was $614-651 \mathrm{bp}$ in size ( 216 bp of the 3 '-end of the 16S rRNA gene, $\sim 328-365$ bp of the ITS region and $\sim 70$ bp of the $5^{\prime}$-end of the $23 \mathrm{~S}$ rRNA gene).

Cloning and sequencing of the ITS region. For each isolate, the amplified ITS region PCR product was directly cloned using the pGEM-T Easy Vector System II (Promega) according to the manufacturer's instructions. Sixteen to twenty-five clones for each strain were selected in order to optimize the likelihood of 'capturing' most, if not all, copies of the ITS region; these were then subjected to further PCR to verify the presence of the insertion (with 614-651 bp insertion) using the primers PGEMT-A and PGEMT-B (Table 2). Eight to fifteen clones for each isolate were directly sent for sequencing; the other clones were screened with the RLB assay (see below) first and only some clones with novel RLB patterns were selected to be sequenced. Cloned PCR products containing the insertion were then purified using the PCR Product Pre-Sequencing kit (USB) and sequenced using the primers PGEMT-A or PGEMT-B with the BigDye Terminator (version 3.1) Cycle Sequencing kit (ABI Prism 373 genetic analyser; Applied Biosystems). After examination of sequence traces of each of the clones, sequences were analysed using the BioManager facility (http://biomanager.info/). Consensus sequences were generated from alignments of sequence data using CLUSTAL W (Thompson et al., 1994) to reconcile ambiguous or conflicting base assignments.

Probe design. ITS sequences obtained as above for the cloned fragments of each of the five species were aligned to determine signature species-specific nucleotide polymorphisms. For each species, more than one assumed species-specific probe was designed targeting different ITS operons: four probes each were for $N$. beijingensis, N. elegans and $N$. thailandica, and three probes each for $N$. blacklockiae and $N$. vinacea (Table 2). Additionally, two previously used actinomycete-specific probes, ACTMY-ITS-a and ACTMY-ITSb (Xiao et al., 2010), were utilized (Table 2) to 'capture' and flag all isolates and cloned ITS PCR products as representing possible 'Nocardia' species. We were unable to devise probes that were specific only for the genus Nocardia.

Altogether, 20 probes were designed. All probes had similar physical characteristics as determined using the Sigma DNA Calculator (http:// 
Table 1. Sequence types, and identification, of cloned PCR products of Nocardia beijingensis, Nocardia blacklockiae, Nocardia elegans, Nocardia thailandica and Nocardia vinacea isolates by ITS sequencing and ITS-based RLB hybridization in comparison with 606 bp 16S rRNA gene sequencing

\begin{tabular}{|c|c|c|c|c|c|}
\hline \multirow[t]{2}{*}{$\begin{array}{l}\text { Strain no. } \\
\text { (identification no.) }\end{array}$} & \multirow{2}{*}{$\begin{array}{l}\text { Identification by } 606 \text { bp 16S rRNA } \\
\text { gene sequencing (GenBank accession } \\
\text { no. of reference strain with } 100 \% \\
\text { sequence similarity) }\end{array}$} & \multicolumn{2}{|c|}{$\begin{array}{l}\text { Identification by ITS spacer } \\
\text { sequencing }\end{array}$} & \multicolumn{2}{|c|}{$\begin{array}{c}\text { Identification by ITS-targeted } \\
\text { RLB assay }\end{array}$} \\
\hline & & Sequence type & $\begin{array}{c}\text { GenBank } \\
\text { accession no. }\end{array}$ & RLB ID & RLB type ${ }^{*}$ \\
\hline \multirow[t]{4}{*}{$1(00-061-3088)$} & N. beijingensis (DQ659901) & N-bei seq I & GQ853473 & N. beijingensis & N-bei RLB I \\
\hline & & N-bei seq II & GQ853474 & N. beijingensis & N-bei RLB I \\
\hline & & N-bei seq III & GQ853472 & N. beijingensis & N-bei RLB I \\
\hline & & N-bei seq IV & GQ853471 & N. beijingensis & N-bei RLB II \\
\hline \multirow[t]{4}{*}{$2(03-294-3356)$} & N. beijingensis (DQ659901) & N-bei seq V & GQ853482 & N. beijingensis & N-bei RLB III \\
\hline & & N-bei seq VI & GQ853481 & N. beijingensis & N-bei RLB III \\
\hline & & N-bei seq VII & GQ853480 & N. beijingensis & N-bei RLB IV \\
\hline & & N-bei seq VIII & GQ853479 & N. beijingensis & N-bei RLB IV \\
\hline \multirow[t]{5}{*}{$3(96-247-0894)$} & N. blacklockiae (EU099360) & N-bla seq I & GQ853494 & N. blacklockiae & N-bla RLB I \\
\hline & & N-bla seq II & GQ853496 & N. blacklockiae & N-bla RLB I \\
\hline & & N-bla seq III & GQ853493 & N. blacklockiae & N-bla RLB II \\
\hline & & N-bla seq IV & GQ853497 & N. blacklockiae & N-bla RLB II \\
\hline & & N-bla seq V & GQ853495 & & N-bla RLB III $\dagger$ \\
\hline \multirow[t]{5}{*}{$4(07-268-4118)$} & N. elegans (DQ659905) & N-ele seq I & GQ853483 & N. elegans & N-ele RLB I \\
\hline & & N-ele seq II & GQ853486 & N. elegans & N-ele RLB I \\
\hline & & N-ele seq III & GQ853485 & N. elegans & N-ele RLB II \\
\hline & & $\mathrm{N}$-ele seq IV & GQ853487 & N. elegans & N-ele RLB II \\
\hline & & N-ele seq V & GQ853484 & N. elegans & N-ele RLB III \\
\hline \multirow[t]{4}{*}{$5(00-355-2649)$} & N. thailandica (AB126874) & N-tha seq I & GQ853476 & N. thailandica & N-tha RLB I \\
\hline & & N-tha seq II & GQ853477 & N. thailandica & N-tha RLB II \\
\hline & & N-tha seq III & GQ853475 & N. thailandica & N-tha RLB III \\
\hline & & N-tha seq IV & GQ853478 & & N-tha RLB IV \\
\hline \multirow[t]{5}{*}{$6(99-146-2360)$} & N. vinacea (DQ659919) & $\mathrm{N}$-vin seq I & GQ853488 & N. vinacea & N-vin RLB I \\
\hline & & $\mathrm{N}$-vin seq II & GQ853491 & N. vinacea & N-vin RLB I \\
\hline & & $\mathrm{N}$-vin seq III & GQ853490 & N. vinacea & N-vin RLB I \\
\hline & & $\mathrm{N}$-vin seq IV & GQ853489 & N. vinacea & $\mathrm{N}$-vin RLB II \\
\hline & & $\mathrm{N}$-vin seq $\mathrm{V}$ & GQ853492 & N. vinacea & N-vin RLB II \\
\hline
\end{tabular}

${ }^{\star}$ See Table 3 for RLB pattern definition.

$\dagger$ The sequence type clone of N-bla seq V failed to hybridize with any of the N. blacklockiae-specific probes but weakly cross-hybridized with the 'Nvin-ITS-c' probe.

$¥$ The sequence type clone of N-tha seq IV failed to hybridize with all $N$. thailandica-specific probes.

www.sigma-genosys.com/calc/dnacalc.asp) (Kong et al., 2006). Probes were $5^{\prime}$-hexylamine-labelled and synthesized by Beijing AuGCT Biotechnology.

PCR/RLB assay. The PCR/RLB assay was evaluated using DNA from cloning experiments as well as from the six isolates (Table 1). PCR for RLB was performed as for the procedure for cloning experiments (see above), with the non-labelled sense primer $16 \mathrm{Sf}$ and the $5^{\prime}$ biotin-labelled antisense primer 23Srb (Table 2). Details for PCR amplification have been described previously (Xiao et al., 2010).

The RLB hybridization assay was performed as reported previously (Kong et al., 2006; Zeng et al. 2007). RLB results were regarded as positive when a hybridization dot signal was clearly visible.

Definition of ITS sequence types (cloned sequences) and RLB pattern types. For every isolate, each cloned ITS sequence (or operon) which demonstrated nucleotide polymorphisms was considered to be a different type for that isolate and assigned as a different 'sequence type' regardless of the magnitude of sequence heterogeneity (Table 1; Supplementary Fig. S1). For RLB results, 'RLB pattern types' were assigned according to the probe hybridization pattern for a given ITS clone or isolate; dissimilar hybridization patterns were considered as different 'RLB pattern types' (see Table 3 and Fig. 1).

\section{RESULTS AND DISCUSSION}

Molecular-based identification of Nocardia species remains a challenge due to the increasing recognition of novel species and taxonomic changes (Brown-Elliott et al., 2006). Given that gene polymorphisms within the conserved $16 \mathrm{~S}$ rRNA gene locus in Nocardia (as for other bacteria) may be 
Table 2. Oligonucleotide primers and probes used in the study

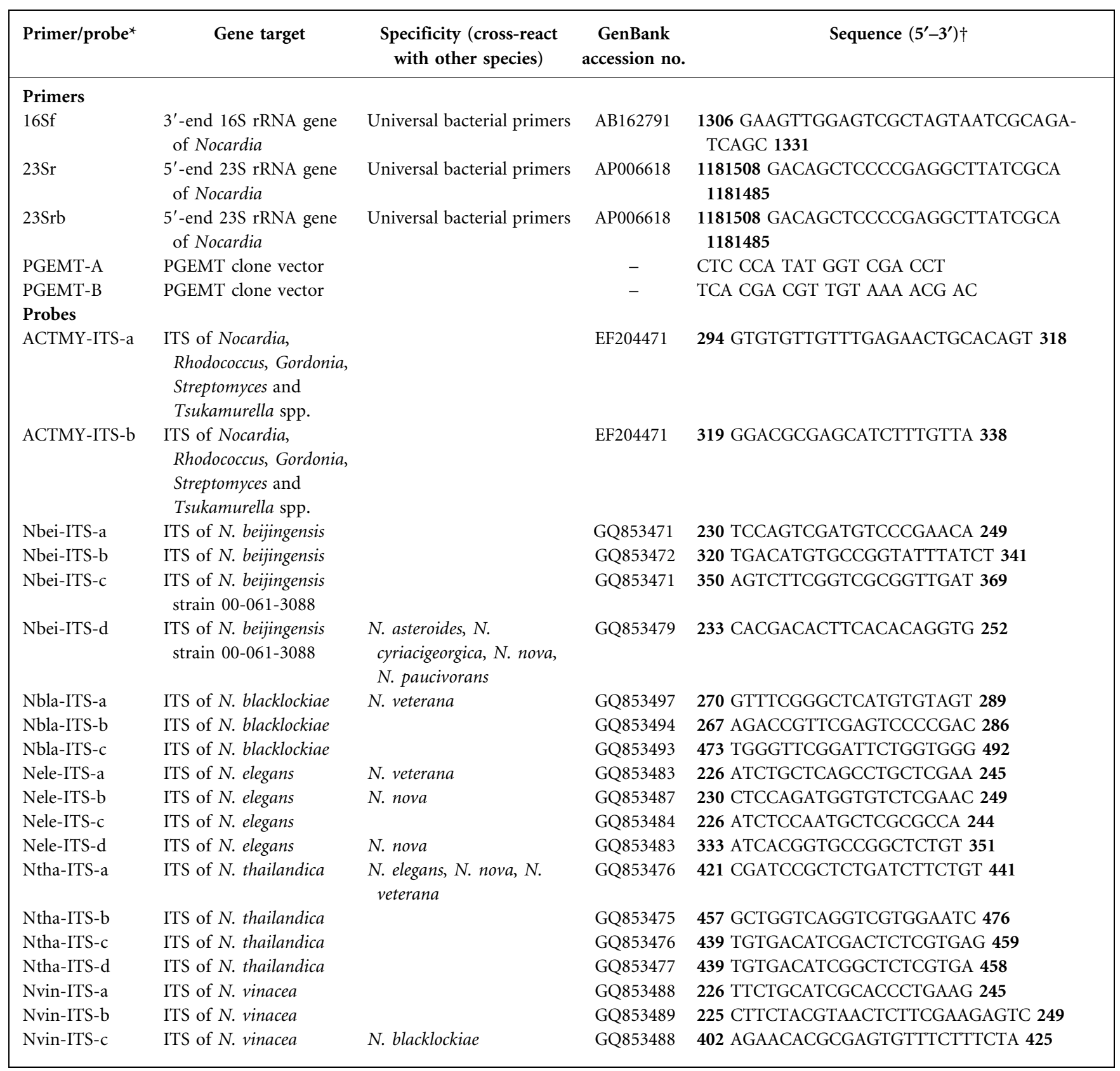

${ }^{*}$ f, Forward; r, reverse; rb, reverse with biotin labelled; ITS, 16S-23S rRNA gene intergenic spacer region.

$\dagger$ Numbers represent the numbered base positions where the primer or probe sequences start or finish (point 1 refer to the corresponding gene GenBank sequence).

insufficient to resolve species identity (Conville et al., 2006; Kong et al., 2009), it is logical to examine the utility of the more variable (bacterial) ITS region for species identification (Boyer et al., 2001; Gurtler \& Stanisich, 1996; Xiao et al., 2010). In this study, we provide accurate sequence data as well as document ITS sequence heterogeneity for five emerging pathogenic Nocardia species. Based on these sequence polymorphisms, we evaluated the ability of an ITS-directed RLB assay to identify the five species. Features of the RLB assay include the high overall sensitivity $(\sim 94 \%)$ and specificity for identification of the target species and its potential as a tool for subtyping of Nocardia.

\section{Sequencing of cloned ITS PCR products and sequence types}

The primers 16Sf and 23Sr (Table 2) amplified the ITS region of all five species (six isolates) targeted by the RLB 
Table 3. RLB pattern types of all target Nocardia isolates and cloned PCR products tested in this study

+ , Presence of hybridization signal.

\begin{tabular}{|c|c|c|c|c|c|c|}
\hline Probe & Species (isolate ID no.) & No. of RLB types & RLB type I & RLB type II & RLB type III & RLB type IV \\
\hline Nbei-ITS-a & $\begin{array}{l}\text { Nocardia beijingensis (00-061-3088 and } \\
03-294-3356)\end{array}$ & 4 & & + & + & \\
\hline Nbei-ITS-b & & & + & & & + \\
\hline Nbei-ITS-c & & & & + & & \\
\hline Nbei-ITS-d & & & + & & & \\
\hline Nbla-ITS-a & Nocardia blacklockiae (96-247-0894) & $3^{*} \dagger$ & & + & & \\
\hline Nbla-ITS-b & & & + & & & \\
\hline Nbla-ITS-c & & & & + & & \\
\hline Nele-ITS-a & Nocardia elegans (07-268-4118) & $3 \dagger$ & + & & & \\
\hline Nele-ITS-b & & & & + & & \\
\hline Nele-ITS-c & & & & & + & \\
\hline Nele-ITS-d & & & + & & & \\
\hline Ntha-ITS-a & Nocardia thailandica (00-355-2649) & 3 & + & & & \\
\hline Ntha-ITS-b & & & & & + & \\
\hline Ntha-ITS-c & & & + & + & & \\
\hline Ntha-ITS-d & & & + & + & & \\
\hline Nvin-ITS-a & Nocardia vinacea (99-146-2360) & 2 & + & & & \\
\hline Nvin-ITS-b & & & & + & & \\
\hline Nvin-ITS-c & & & + & & & \\
\hline
\end{tabular}

*One sequence type of $N$. blacklockiae (N-bla seq V in Table 1) failed to hybridize with any of the N. blacklockiae-specific probes but crosshybridized with the Nvin-ITS-c probe.

$\dagger$ Cross-hybridization signal not shown in the table (see Fig. 1 for details).

assay. The expected PCR product of $\sim 614-651 \mathrm{bp}$ was obtained in each instance. One hundred and eight successful clones were obtained in this study: 15 and 12 clones for the two strains of $N$. beijingensis, and 25, 18, 22 and 16 clones for N. blacklockiae, N. elegans, N. thailandica and $N$. vinacea, respectively. All clones were examined by RLB assay (see below).

Seventy of the 108 successful clones were sequenced, including 15 and 12 clone sequences for the two strains of $N$. beijingensis, and 12, 14, 8 and 9 clone sequences for $N$. blacklockiae, $N$. elegans, $N$. thailandica and $N$. vinacea, respectively. Comparison of the sequences initially revealed eight different ITS sequences or sequence types for the two $N$. beijingensis strains (four sequence types each), four sequence types each for strains of $N$. blacklockiae, N. elegans and $N$. vinacea, and three sequence types for the $N$. thailandica isolate (Table 1). The sites of ITS sequence polymorphisms are detailed in Supplementary Fig. S1. However, after RLB analysis, a further sequence type for each of $N$. blacklockiae and $N$. thailandica was identified (see below); thus a total of five and four ITS types were evident for these species, respectively (Table 1 ).

The finding of more than one ITS type or operon within a single Nocardia isolate for each of the study species is similar to that observed for the 16S rRNA operons, particularly in isolates of $N$. nova (Conville \& Witebsky, 2005 , 2007). Multiple copy numbers of operons containing the genes encoding all of the $16 \mathrm{~S}$ rRNA gene, $23 \mathrm{~S}$ rRNA gene and the ITS regions have also been described for other bacteria including Escherichia coli (seven operons) and Clostridium difficile (11 operons) (Hashimoto et al., 2003; Sadeghifard et al., 2006). Such ITS sequence heterogeneity may compromise direct ITS sequence-based identification of most, if not all, Nocardia species. Therefore, alternative methods exploiting polymorphisms within this locus warrant further study.

\section{Probe development}

Alignment of ITS sequences obtained from sequencing of cloned PCR products revealed three short blocks of sequence dissimilarity - nucleotide positions 231-304 bp, 328-385 bp and 409-530 bp - between species (see Supplementary Fig. S1), which were variably present according to the clone or sequence type. Additional species-specific single nucleotide polymorphisms were also present. All these sites of nucleotide variation were used as the basis for probe design to differentiate between and within species (Table 2; Supplementary Fig. S1). Since there were a number of sequence types for each of the species (Table 1), multiple (three to four) sequence type- or operon-specific probes (all also designed to be assumed species-specific) were devised for each species. These probes were then tested for their ability to identify the target species individually and in combination with other probes. 


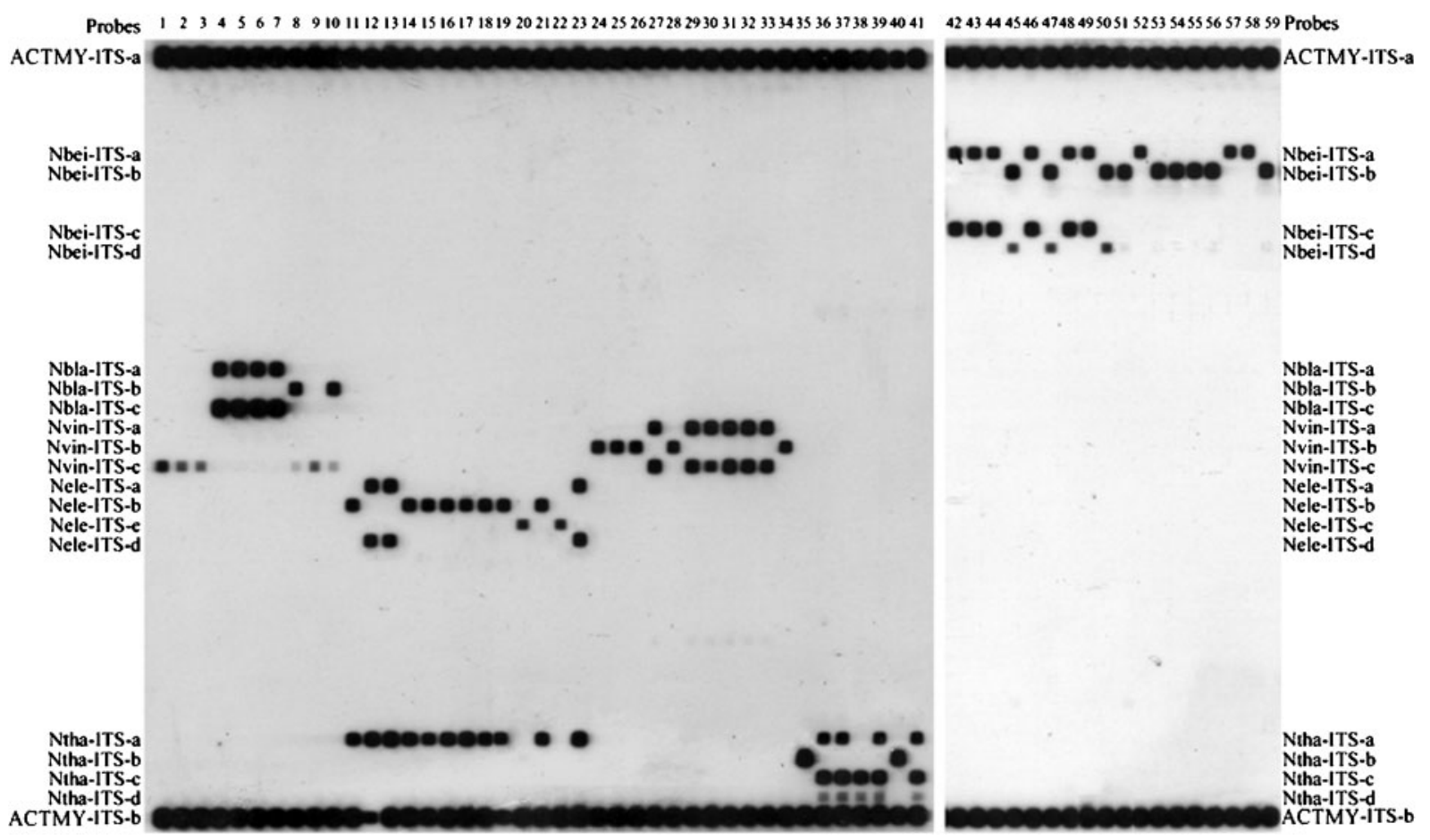

Fig. 1. RLB hybridization results for cloned PCR products of the five emerging Nocardia species. Probe designations are given on the left and right side of the figure. For designations of RLB pattern type, please refer to Table 3. Lanes 1-10, clones of strain 96-247-0894 (N. blacklockiae): 1-3 and 9, N-bla RLB III; 4-7, N-bla RLB II; 8 and 10, N-bla RLB I. Lanes 11-23, clones of strain 07-268-4118 (N. elegans): 11, 14-19 and 21, N-ele RLB II; 12, 13 and 23, N-ele RLB I; 20 and 22, N-ele RLB III. Lanes 24-34, clones of strain 99-146-2361 (N. vinacea): 24-26, 28 and 34, N-vin RLB II; 27 and 29-33, N-vin RLB I. Lanes 35-41, clones of strain 00-355-2649 (N. thailandica): 35 and 40, N-tha RLB III; 36, 37, 39 and 41, N-tha RLB I; 38, N-tha RLB II. Lanes 42-50, clones of strain 00-061-3088 (N. beijingensis): 42-44, 46, 48 and 49, N-bei RLB II; 45,47 and 50, N-bei RLB I. Lanes 51-59, clones of strain 03-294-3356 ( $N$. beijingensis): 51, 53-56 and 59, N-bei RLB IV; 52,57 and 58, N-bei RLB III.

\section{Identification of cloned PCR products and Nocardia isolates by the RLB assay and RLB- assisted identification of new sequence types}

The two actinomycete-specific probes ACTMY-ITS-a and ACTMY-ITS-b hybridized with cloned PCR products containing the expected $\sim 614-651 \mathrm{bp}$ insertion and with DNA from the six isolates. These products were thus flagged as representing possible 'Nocardia species'.

By use of multiple species/operon-specific probes designed for the study (Table 2), the majority (102 of 108 or $94.4 \%$ ) of clones from all six isolates were correctly identified by the RLB assay (Table 1). However, four of 25 cloned products of N. blacklockiae (strain 96-247-0894) produced no RLB signal with all Nbla-ITS-probes. Instead, they only weakly cross-hybridized with the Nvin-ITS-c probe (Fig. 1, lanes $1-3$ and 9 and see 'Specificity of the RLB assay'). Examination of the entire ITS locus revealed that they contained novel sequence regions, representing an additional and further sequence type for N. blacklockiae (N-bla seq V, GenBank accession no. GQ853495; Table 1), and thus were not able to hybridize with the N. blacklockiae- specific probes as intended. Two of 22 clones of $N$. thailandica produced no RLB signal with the $N$. thailandica probes (data not shown); their sequences also represented a new sequence type (N-tha seq IV, GenBank accession no. GQ853478; Table 1). All six isolates (Table 1) also underwent RLB analysis and were correctly identified by the assay (see below for RLB pattern types).

The finding of additional ITS sequence types of bacteria using a probe-based approach is consistent with the report by Sadeghifard et al. (2006) of the mosaic nature of $C$. difficile ITS operons and their hypothesis that further characterization of ITS sequence by oligonucleotide arrays is likely to uncover new sequence types. Our 'macro-array' RLB assay was similarly able to identify six new ITS types, demonstrating the potential of probe-based formats for screening for novel ITS (or indeed other gene loci) sequence types.

\section{RLB pattern types}

A number of unique RLB pattern types, as determined from the pattern of hybridization with specific probes, were 
observed for the cloned products of each of N. beijingensis, $N$. blacklockiae, $N$. elegans, $N$ thailandica and $N$. vinacea (range two to four RLB types per species; Tables 1 and 3, Fig. 1), with the exception of six clones described above that contained new ITS sequence types. For a single isolate, overall there were fewer RLB pattern types compared with sequence types, except for the $N$. thailandica strain, where each ITS sequence type also exhibited a different RLB type (Table 1).

Direct RLB analysis of the isolates yielded RLB patterns which were, in general, a composite of the patterns of their sequence types. The isolates hybridized with all of their respective species-specific probes, with the exception of $N$. beijingensis strain 03-294-3352, which hybridized only with Nbei-ITS-a and Nbei-ITS-b. We were limited by our access to only single strains of the target species (two strains for $N$. beijingensis); analysis of a larger number of isolates is required to determine the wider application of the RLB assay in the identification of these species.

\section{Specificity of the RLB assay}

The specificity of the RLB assay was tested against isolates of the five species of interest (target species; Table 3 ) as well as a panel of 135 non-target Nocardia isolates (14 species) (see Supplementary Table S1). All N. beijingensis-ITS, N. blacklockiae-ITS and N. elegans-ITS probes were highly specific where the five target species were evaluated. However, cross-hybridization was observed between (i) the Nvin-ITS-c probe and four N. blacklockiae clones and (ii) the Ntha-ITS-a probe and N. elegans DNA (11 clones) (Fig. 1). When tested on non-target Nocardia species, RLB analysis found that three of the $N$. elegans-specific probes hybridized with some strains of $N$. nova and Nocardia veterana and the Nbei-ITS-d probe cross-hybridized with certain strains of Nocardia asteroides and N. cyriacigeorgica (Table 2; data for individual strains not shown). It is possible that these species may share similar ITS operon sequences, at least in the region used for probe design.

Another key finding of the present study was that the two $N$. beijingensis strains exhibited different ITS-targeted RLB patterns. This supports our recent observation of hybridization of different, unique $N$. farcinica strains with different ITS-based RLB probes (Xiao et al., 2010) and suggests the potential of the RLB format as a tool for molecular subtyping of Nocardia species. Further study of large numbers of strains representing a wider range of species is required to improve the design of species/ operon-specific probes with sufficient discriminatory capability (Sadeghifard et al., 2006; Park et al., 2005).

Because of the diverse range of nocardial pathogens, DNA sequencing has become the preferred method for definitive species determination. We confirm its accuracy of $16 \mathrm{~S}$ rRNA gene sequencing (Kong et al., 2009) in this capacity to identify five emerging Nocardia species. However, this approach is expensive (AUD15/USD14 per isolate) and limited by the quality of sequence databases (Kong et al., 2009; Woo et al., 2009). Moreover, the presence of multiple ITS operons, as shown in this study, has the potential to lead to misidentifications, at least for the five species analysed. Probe-based hybridization technologies have been successful in differentiating Mycobacteria species (Park et al., 2005; Xiong et al., 2006) but their utility for Nocardia identification is not well-established (Alfaresi \& Elkosh, 2006). The RLB assay allowed simultaneous analysis of multiple isolates against multiple probes, demonstrated comparable sensitivity to ITS sequencing and may be a useful adjunct to sequencing for species identification of the five target species. Although only 20 probes were utilized in the present study, up to 43 can be incorporated, extending the range of species or sequence types that can be identified.

In conclusion, the present study has for the first time provided, through cloning experiments, accurate ITS sequence data for the emerging species $N$. beijingensis, $N$. blacklockiae, $N$. elegans, $N$. thailandica and $N$. vinacea. Multiple ITS sequence types or operons were evident within a single isolate for each species. The incorporation of species/operon-specific probes into a RLB assay enabled the identification of unique RLB patterns for each of the species. The PCR/RLB assay showed promise both in the identification and genotyping of these five Nocardia species. More detailed studies of the polymorphism within the ITS locus of other Nocardia species may further advance our capacity to reliably identify and subtype medically important Nocardia species.

\section{ACKNOWLEDGEMENTS}

We thank the staff of the Westmead Millennium Institute sequencing laboratory, Sydney, for performing the sequencing.

\section{REFERENCES}

Alfaresi, M. \& Elkosh, A. (2006). Rapid identification of clinically relevant Nocardia species using real-time PCR with SYBR Green and melting-curve analysis. J Med Microbiol 55, 1711-1715.

Boyer, S. L., Flechtner, V. R. \& Johansen, J. R. (2001). Is the 16S-23S rRNA internal transcribed spacer region a good tool for use in molecular systematics and population genetics? A case study in cyanobacteria. Mol Biol Evol 18, 1057-1069.

Brown-Elliott, B. A., Brown, J. M., Conville, P. S. \& Wallace, R. J., Jr (2006). Clinical and laboratory features of the Nocardia spp. based on current molecular taxonomy. Clin Microbiol Rev 19, 259-282.

Conville, P. S. \& Witebsky, F. G. (2005). Multiple copies of the $16 \mathrm{~S}$ rRNA gene in Nocardia nova isolates and implications for sequencebased identification procedures. J Clin Microbiol 43, 2881-2885.

Conville, P. S. \& Witebsky, F. G. (2007). Analysis of multiple differing copies of the $16 \mathrm{~S}$ rRNA gene in five clinical isolates and three type strains of Nocardia species and implications for species assignment. J Clin Microbiol 45, 1146-1151.

Conville, P. S., Zelazny, A. M. \& Witebsky, F. G. (2006). Analysis of secAl gene sequences for identification of Nocardia species. J Clin Microbiol 44, 2760-2766. 
Conville, P. S., Brown, J. M., Steigerwalt, A. G., Brown-Elliott, B. A. \& Witebsky, F. G. (2008). Nocardia wallacei sp. nov. and Nocardia blacklockiae sp. nov., human pathogens and members of the "Nocardia transvalensis Complex". J Clin Microbiol 46, 1178-1184.

Gurtler, V. \& Stanisich, V. A. (1996). New approaches to typing and identification of bacteria using the 16S-23S rDNA spacer region. Microbiology 142, 3-16.

Hashimoto, J. G., Stevenson, B. S. \& Schmidt, T. M. (2003). Rates and consequences of recombination between rRNA operons. J Bacteriol 185, 966-972.

Kageyama, A., Poonwan, N., Yazawa, K., Mikami, Y. \& Nishimura, K. (2004a). Nocardia beijingensis, is a pathogenic bacterium to humans: the first infectious cases in Thailand and Japan. Mycopathologia 157, $155-161$.

Kageyama, A., Poonwan, N., Yazawa, K., Suzuki, S., Kroppenstedt, R. M. \& Mikami, Y. (2004b). Nocardia vermiculata sp. nov. and Nocardia thailandica sp. nov. isolated from clinical specimens. Actinomycetologica 18, 27-33.

Kageyama, A., Yazawa, K., Nishimura, K. \& Mikami, Y. (2005). Nocardia anaemiae sp. nov. isolated from an immunocompromised patient and the first isolation report of Nocardia vinacea from humans. Nippon Ishinkin Gakkai Zasshi 46, 21-26.

Kong, F., Brown, M., Sabananthan, A., Zeng, X. \& Gilbert, G. L. (2006). Multiplex PCR-based reverse line blot hybridization assay to identify 23 Streptococcus pneumoniae polysaccharide vaccine serotypes. J Clin Microbiol 44, 1887-1891.

Kong, F., Chen, S. C. A., Chen, X., Sintchenko, V., Halliday, C., Cai, L., Tong, Z., Lee, O. C. \& Sorrell, T. C. (2009). Assignment of reference $16 \mathrm{~S}$ rDNA sequences, and species-specific sequence polymorphisms improves species identification of clinically-important Nocardia. Open Microbiol J 3, 97-105.

Luong, M. S., Bret, C., Godreuil, S., Terru, D., Chong, G., Marchandin, H. \& Mourad, G. (2009). Pyoderma vegetans in a renal transplant recipient: first case of human infection with Nocardia vinacea. Transplantation $\mathbf{8 7}$ 1898-1899.

McNeil, M. M. \& Brown, J. M. (1994). The medically important aerobic actinomycetes: epidemiology and microbiology. Clin Microbiol Rev 7, 357-417.

Park, H., Jang, H., Song, E., Chang, C. L., Lee, M., Jeong, S., Park, J., Kang, B. \& Kim, C. (2005). Detection and genotyping of Mycobacterium species from clinical isolates and specimens by oligonucleotide array. J Clin Microbiol 43, 1782-1788.

Rodriguez-Nava, V., Couble, A., Devulder, G., Flandrois, J. P., Boiron, P. \& Laurent, F. (2006). Use of PCR-restriction enzyme pattern analysis and sequencing database for $h s p 65$ gene-based identification of Nocardia species. J Clin Microbiol 44, 536-546.
Roth, A., Andrees, S., Kroppenstedt, R. M., Harmsen, D. \& Mauch, H. (2003). Phylogeny of the genus Nocardia based on reassessed $16 \mathrm{~S}$ rRNA gene sequences reveals underspeciation and division of strains classified as Nocardia asteroides into three established species and two unnamed taxons. J Clin Microbiol 41, 851-856.

Sadeghifard, N., Gürtler, V., Beer, M. \& Seviour, R. J. (2006). The mosaic nature of intergenic 16S-23S rRNA spacer regions suggests rRNA operon copy number variation in Clostridium difficile strains. Appl Environ Microbiol 72, 7311-7323.

Saubolle, M. A. \& Sussland, D. (2003). Nocardiosis: review of clinical and laboratory experience. J Clin Microbiol 41, 4497-4501.

Su, S. C., Vaneechoutte, M., Dijkshoorn, L., Wei, Y. F., Chen, Y. L. \& Chang, T. C. (2009). Identification of non-fermenting Gram-negative bacteria of clinical importance by an oligonucleotide array. $J$ Med Microbiol 58, 596-605.

Takeda, K., Kang, Y., Yazawa, K., Gonoi, T. \& Mikami, Y. (2010). Phylogenetic studies of Nocardia species based on gyrB gene analyses. J Med Microbiol 59, 165-171.

Thompson, J. D., Higgins, D. G. \& Gibson, T. J. (1994). ClUSTAL W: improving the sensitivity of progressive multiple sequence alignment through sequence weighting, position-specific gap penalties and weight matrix choice. Nucleic Acids Res 22, 4673-4680.

Wang, Y., Kong, F., Gilbert, G. L., Brown, M., Gao, W., Yu, S. \& Yang, Y. (2008). Use of a multiplex PCR-based reverse line blot (mPCR/RLB) hybridisation assay for the rapid identification of bacterial pathogens. Clin Microbiol Infect 14, 155-160.

Woo, P. C., Teng, J. L., Wu, J. K., Leung, F. P., Tse, H., Fung, A. M., Lau, S. K. \& Yuen, K. Y. (2009). Guidelines for interpretation of $16 \mathrm{~S}$ rRNA gene sequence-based results for identification of medically important aerobic Gram-positive bacteria. J Med Microbiol 58, 1030-1036.

Xiao, M., Kong, F., Sorrell, T. C., Cao, Y., Lee, O. C., Liu, Y., Sintchenko, V. \& Chen, S. C. A. (2010). Identification of pathogenic Nocardia species by reverse line blot hybridization targeting the $16 \mathrm{~S}$ rRNA and 16S-23S rRNA gene spacer regions. J Clin Microbiol 48, 503-511.

Xiong, L., Kong, F., Yang, Y., Cheng, J. \& Gilbert, G. L. (2006). Use of PCR and reverse line blot hybridization macroarray based on 16S-23S rRNA gene internal transcribed spacer sequences for rapid identification of 34 Mycobacterium species. J Clin Microbiol 44, 3544-3550.

Yassin, A. F. \& Brenner, S. (2005). Nocardia elegans sp. nov., a member of the Nocardia vaccinii clade isolated from sputum. Int J Syst Evol Microbiol 55, 1505-1509.

Zeng, X., Kong, F., Halliday, C., Chen, S., Lau, A., Playford, G. \& Sorrell, T. C. (2007). Reverse line blot hybridization assay for identification of medically important fungi from culture and clinical specimens. J Clin Microbiol 45, 2872-2880. 